\title{
Objective Rating of the Launch Behavior of Conventional, Hybrid and Electric Vehicles
}

\author{
Christian Dorsch ${ }^{1}\left[\right.$ Xiao Wang $^{1} \cdot$ Ferit Küçükay ${ }^{1}$
}

Received: 27 April 2020 / Accepted: 11 December 2020 / Published online: 27 January 2021

(c) The Author(s) 2021

\begin{abstract}
The calibration of conventional, hybrid and electric drivetrains is an important process during the development phase of any vehicle. Therefore, to optimize the comfort and dynamic behavior (known as driveability), many test drives are performed by experienced drivers during different driving maneuvers, e.g., launch, re-launch or gear shift. However, the process can be kept more consistent and independent of human-based deviations by using objective ratings. This study first introduces an objective rating system developed for the launch behavior of conventional vehicles with automatic transmission, dualclutch transmission, and alternative drivetrains. Then, the launch behavior, namely comfort and dynamic quality, is compared between two conventional vehicles, a plug-in hybrid electric vehicle and a battery electric vehicle. Results show the benefits of pure electric drivetrains due to the lack of launch and shifting elements, as well as the usage of a highly dynamic electric motor. While the plug-in hybrid achieves a $10 \%$ higher overall rating compared to the baseline conventional vehicle, the pure electric vehicle even achieves a $21 \%$ higher overall rating. The results also highlight the optimization potential of battery electric vehicles regarding their comfort and dynamic characteristics. The transitions and the gradient of the acceleration build-up have a major influence on the launch quality.
\end{abstract}

Keywords Objective rating $\cdot$ Launch behavior $\cdot$ Driveability $\cdot$ Transmission calibration · Drivetrain calibration

$\begin{array}{ll}\text { Abbreviations } \\ \text { AWD } & \text { All-wheel drive } \\ \text { BEV } & \text { Battery electric vehicle } \\ \text { DCT } & \text { Dual-clutch transmission } \\ \text { ECU } & \text { Electronic control unit } \\ \text { EM } & \text { Electric motor } \\ \text { FWD } & \text { Front-wheel drive } \\ \text { ICE } & \text { Internal combustion engine } \\ \text { ICV } & \text { Internal combustion engine vehicle } \\ \text { PHEV } & \text { Plug-in hybrid electric vehicle }\end{array}$

\section{Introduction}

Investigations on the driveability, especially of passenger cars, have been conducted for decades in the automotive industry. There are many different operation modes to be assessed: e.g., idle, engine start, tip in, tip out, launch and

\footnotetext{
Christian Dorsch

c.dorsch@tu-bs.de

1 Institute of Automotive Engineering, Technische Universität Braunschweig, Braunschweig 38106, Germany
}

gear shifts [1]. Often, these tests are carried out by professionals with a high level of experience. Demanded by the increasing complexity and diversity of powertrains, suspensions, steering and brake systems, the research on objectively characterizing the behavior of a vehicle has been widely studied [1-6]. Different tools and methods have been developed and applied [7-10]. While earlier studies were focusing on the impact of the internal combustion engine (ICE) and its exhaust after-treatment systems [11], newer studies investigate all kinds of potential effects created by drivetrain components and their control strategies (e.g., transmissions and electric motors). Nowadays, objective rating systems are used for motorcycles [12], full-hybrids [13, 14] as well as commercial heavy-duty trucks $[15,16]$. The main goal of these approaches is objectively identifying the optimization potentials in the hardware and control solutions to improve the driving experience. In order to reduce costs and test duration, it is helpful to reduce the number of measurements performed on a test track or in real traffic situations. Roadto-rig solutions [17] or simulations [18] appear to be very promising. When using these methods, calibration engineers cannot rely on their experience to evaluate the driveability since important physical information is not perceptible (e.g., 
longitudinal, lateral and vertical acceleration). Therefore, a robust and reliable objective rating system is required to transfer calibration work to test rigs and simulation tools.

The launch of a vehicle (also called drive-off, drive away or take-off) is critical in driveability assessments. It marks the transition from standstill to moving. There are usually no external sources of excitation such as vibrations due to road conditions or airstream. Thus, only the powertrain and its calibration are responsible for the perceived quality of the launch. For conventional and add-on hybrid vehicles, the combination of engine and launch element (e.g. clutch or torque converter) are the most influential components. The provision of torque at the crankshaft output during the highly dynamic launch phase is crucial, especially considering technologies like start-stop and turbochargers. Simulation models have been developed to calculate this transient behavior [19-21]. Manual transmissions require the driver to control the engagement of the clutch. Therefore, the driver has a higher responsibility for the quality of the launch. With increasing application of dual-clutch transmissions (DCTs), the demand for optimal calibration of the clutch actuation during the launch is growing [22]. Hybrid and electric vehicles offer many new opportunities for optimizing the driveability, especially the launch quality. There are dozens of possible topologies and configurations [23, 24], each with its own advantages and disadvantages regarding performance, efficiency and driveability [25]. Battery electric vehicles (BEVs) and some plug-in hybrid electric vehicles (PHEVs) are usually considered to have a better launch quality due to their electric motor (EM) and the absence of launch elements. This study investigates the launch quality of four different drivetrains (including two internal combustion engine vehicles (ICVs), one PHEV and one BEV) and objectively quantifies the perceived quality of multiple launch maneuvers.

\section{Objective Rating System}

Subjective ratings given by experienced engineers build the foundation for all calibration processes [26]. These ratings will be recorded by the calibration engineer to evaluate the process. The basic idea of objective rating systems is to find physical and measurable key parameters that highly correlate with the subjective ratings. These correlations are used during a multiple linear regression analysis to find a suitable function that allows giving an objective rating. Figure 1 shows the method used: first, test drives have to be performed in order to obtain measured data and corresponding subjective ratings. Afterward, this information has to be matched and transformed into an objective rating system. The rating system for launch maneuvers used in this study is based on the work presented in Refs. [27] and [28].

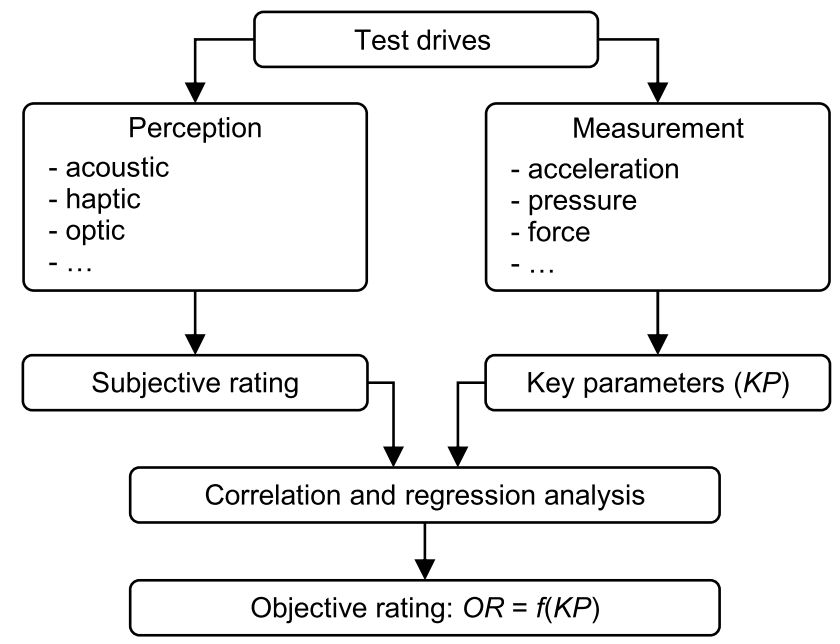

Fig. 1 Method of objectification based on subjective ratings and measurement data [27]

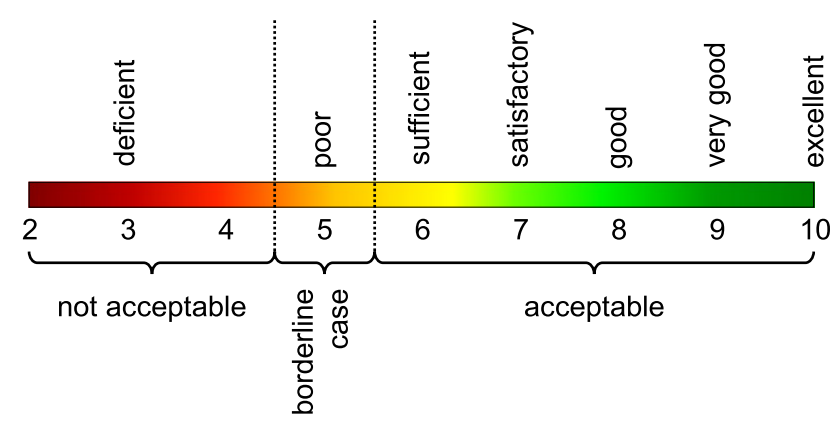

Fig. 2 Rating scale including numeric ratings, color scheme and verbal expressions

\subsection{Rating Scale}

This objectification method uses an adapted version of the rating scale introduced in Ref. [29]. It has been used and modified in many investigations in the automotive industry [30,31]. In this study, it ranges from 2 (worst result possible) to 10 (best result possible). Figure 2 presents the color scheme that is used to visualize the results (see Sect. 4). Red indicates a low rating, while green indicates a high rating. The verbal expressions are used to introduce the rating system to new test drivers so that they have a better understanding of the numeric values. Objective ratings higher than 5 are acceptable for application in series cars while lower ratings are not acceptable in series application. Objective ratings of 5 represent a borderline case.

\subsection{Key Parameters and Weighted Ratings}

To calculate the objective ratings, key parameters have to be identified from the measured data. This study uses 
partially modified key parameters presented in Refs. [27, 28]. In total, there are four key parameters: launch jerk $K P_{\text {jer }}$, overall vibrations $K P_{\text {vib }}$, response delay time $K P_{\text {del }}$ and distance traveled in $3 \mathrm{~s} K P_{\text {dis. }}$. The first two make up a comfort rating while the last two make up a dynamic rating. The key parameters are used in Eqs. (1)-(4) to calculate their objective ratings $\left(O R_{\text {jer }}, O R_{\text {vib }}, O R_{\text {del }}\right.$ and $O R_{\text {dis }}$ ). The variables $c_{1,1}$ to $c_{1,4}$ are used as offsets, while the variables $c_{2,1}$ to $c_{2,4}$ represent constant factors to the key parameters.

$O R_{\text {jer }}=c_{1,1}+c_{2,1} \cdot K P_{\text {jer }}$

$O R_{\mathrm{vib}}=c_{1,2}+c_{2,2} \cdot K P_{\mathrm{vib}}$

$O R_{\text {del }}=c_{1,3}+c_{2,3} \cdot K P_{\text {del }}$

$O R_{\text {dis }}=c_{1,4}+c_{2,4} \cdot K P_{\text {dis }}$

The values of $c_{1, x}$ and $c_{2, x}$ are the result of the correlation and regression analysis described in Fig. 1, respectively. The complete process, including information about the on-road investigations executed by multiple test drivers, is described in detail in Ref. [27]. Figure 3 gives an overview of the four key parameters for an exemplary launch maneuver. They are described in Sects. 2.2.1-2.2.4.

The observation period begins when the accelerator pedal is pressed at $t_{1}$ and ends after an additional $3 \mathrm{~s}\left(t_{4}\right)$.

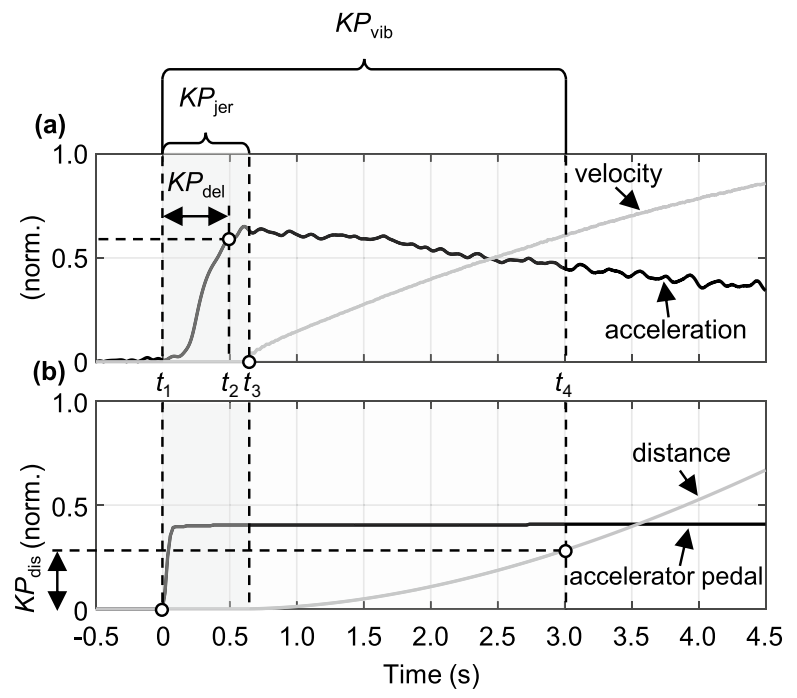

Fig. 3 Key parameters and characteristic time steps of an exemplary launch maneuver for (a) normalized acceleration and velocity and (b) normalized distance and accelerator pedal

\subsubsection{Launch Jerk}

The first sensible shock before the vehicle starts moving (velocity $>0$ at $t_{3}$ in Fig. 3 ) is considered as the launch jerk. The corresponding key parameter $K P_{\text {jer }}$ is defined as the root mean square of the 1 to $10 \mathrm{~Hz}$ band filtered acceleration signal $a_{1 \mathrm{~Hz}-10 \mathrm{~Hz}}$.

$K P_{\text {jer }}=\sqrt{\frac{1}{t_{3}-t_{1}} \int_{t_{1}}^{t_{3}} a_{1 \mathrm{~Hz}-10 \mathrm{~Hz}}(t) \mathrm{d} t}$

Between $t_{1}$ and $t_{3}$, the launch element in the powertrain starts to transfer torque. The elasticities have to be overcome [27].

\subsubsection{Overall Vibrations}

The time span between $t_{1}$ and $t_{4}$ is relevant to the overall vibrations while the vehicle is moving. The key parameter $K P_{\text {vib }}$ is defined as the root mean square of the 2 to $10 \mathrm{~Hz}$ band filtered acceleration signal $a_{2 \mathrm{~Hz}-10 \mathrm{~Hz}}$.

$K P_{\text {vib }}=\sqrt{\frac{1}{t_{4}-t_{1}} \int_{t_{1}}^{t_{4}} a_{2 \mathrm{~Hz}-10 \mathrm{~Hz}}(t) \mathrm{d} t}$

In previous investigations, $t_{4}$ was defined as the synchronous point between engine crankshaft speed and transmission input shaft speed $[27,28]$. Since in this study BEVs without separating clutches are also considered, the criteria for $t_{4}$ have been revised. In order to achieve comparable results, the end point is set to a fixed value of $3 \mathrm{~s}$ after the accelerator pedal is pressed.

\subsubsection{Response Delay Time}

When pressing the accelerator pedal, there is a time delay before the acceleration reaches a saturated level, which happens at $t_{2}$ in Fig. 3. The key parameter $K P_{\text {del }}$ is defined as the time difference between $\mathrm{t}_{2}$ and $\mathrm{t}_{1}$.

$K P_{\text {del }}=t_{2}-t_{1}$

To identify $t_{2}$, the maxima of the $10 \mathrm{~Hz}$ low-pass-filtered acceleration signal $a_{10 \mathrm{~Hz}, \mathrm{low}}$ have to be analyzed. A saturated level of acceleration is assumed if the current local maximum plus $20 \%$ of its value is higher or equal to the global maximum (between $t_{1}$ and $t_{4}$ ).

$t_{2}=t\left(1.2 \cdot \max _{\text {local }} a_{10 \mathrm{~Hz}, \text { low }} \geq \max _{\text {global }} a_{10 \mathrm{~Hz}, \text { low }}\right)$ 


\subsubsection{Distance Traveled}

The last key parameter is $K P_{\text {dis }}$. It is calculated by integrating the vehicle speed $v$ for the first $3 \mathrm{~s}$ after pressing the accelerator pedal. In order to include the actual vehicle data into the rating, the torque to weight ratio $T W R$ of the vehicle is also considered.

$K P_{\text {dis }}=\int_{t_{1}}^{t_{4}} v \mathrm{~d} t \cdot \frac{1}{T W R}$

Higher vehicle mass $m$ and/or lower available maximum torque at the transmission input shaft $T_{\text {ICE/EM,max }}$ lead to a higher $K P_{\text {dis }}$ and therefore to a higher objective ratings. This helps to take the expectations of the vehicle's performance into account.

$K P_{\mathrm{dis}}=\int_{t_{1}}^{t_{4}} v \mathrm{~d} t \cdot \frac{m}{T_{\mathrm{ICE} / \mathrm{EM}, \max }}$

\subsubsection{Weightings for Comfort, Dynamic and Overall Rating}

After the objective ratings for each key parameter have been determined, they are transferred into comfort and dynamic ratings. As shown in Fig. 4, the objective ratings $O R_{\text {jer }}$ and $O R_{\text {vib }}$ are combined to form $O R_{\mathrm{c}}$, while $O R_{\text {del }}$ and $O R_{\text {dis }}$ are combined to form $O R_{\mathrm{d}}$.

The merging of the ratings depends on the weighting coefficients $w_{\mathrm{c}}, w_{\mathrm{d}}$ and $w_{\mathrm{o}}$. The values of these coefficients vary between 0 and 1 .

$$
\begin{aligned}
& O R_{\mathrm{c}}=w_{\mathrm{c}} \cdot O R_{\mathrm{jer}}+\left(1-w_{\mathrm{c}}\right) \cdot O R_{\mathrm{vib}} \\
& O R_{\mathrm{d}}=w_{\mathrm{d}} \cdot O R_{\mathrm{del}}+\left(1-w_{\mathrm{d}}\right) \cdot O R_{\mathrm{dis}} \\
& O R_{\mathrm{o}}=w_{\mathrm{o}} \cdot O R_{\mathrm{c}}+\left(1-w_{\mathrm{o}}\right) \cdot O R_{\mathrm{d}}
\end{aligned}
$$

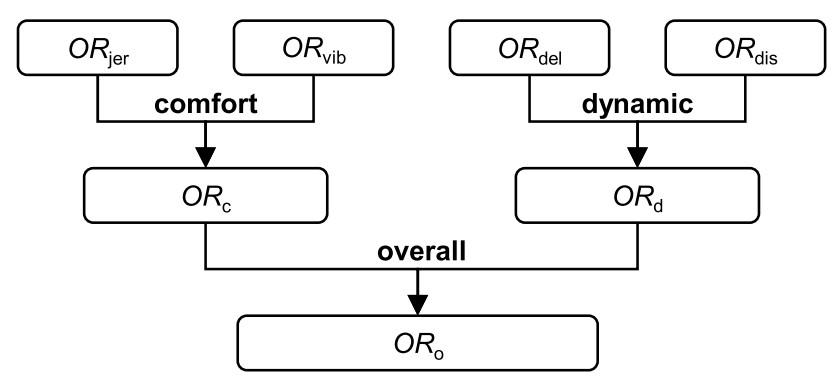

Fig. 4 Merging of the objective ratings into comfort, dynamic and overall ratings
The weighting coefficients $w_{\mathrm{c}}$ and $w_{\mathrm{d}}$ depend only on the accelerator pedal position $A P P$, while $w_{\mathrm{o}}$ is additionally affected by the power to weight ratio of the vehicle.

$w_{\mathrm{c}}=f(A P P)$

$w_{\mathrm{d}}=f(A P P)$

$w_{\mathrm{o}}=f(A P P, P W R)$

With increasing accelerator pedal positions, the values of $w_{\mathrm{c}}, w_{\mathrm{d}}$ and $w_{\mathrm{o}}$ are decreasing. Vehicles with higher power to weight ratio will receive lower values for $w_{\mathrm{o}}$ to meet the expectations on "sporty" cars. The weighting coefficients have been identified in Refs. [27, 28]. The results allow a comparison between multiple cars from different segments and powertrain classes. For example, a small A-segment vehicle (as defined by the European Commission [32]) with low power output could achieve the same overall rating compared to a sporty S-segment vehicle with higher power output. The rating system has been developed primarily for conventional powertrains using automatic transmission and dual-clutch transmission (DCT). Since the system does not require any specific signals or data that are exclusively limited to these powertrain configurations, it may also be used to investigate the launch behavior of hybrid and pure electric vehicles.

\subsection{Usage of the Rating System}

The rating system may be used for multiple purposes. Some of these purposes are A-B comparisons (like presented in this study), documentation and optimization processes.

\subsubsection{A-B Comparison}

Comparing different vehicle classes, drivetrain systems or electronic control unit (ECU) calibration versions while keeping a standardized rating behavior is the key benefit of an objective rating system. Since the evaluation is based on technical parameters, the rating is more consistent and repeatable compared to a human-based subjective rating. As shown in this study, the rating system may be used to identify the unique behavior of different drivetrain systems. It could also be used to evaluate different ECU calibration versions during the development process of a vehicle.

\subsubsection{Documentation of Calibration Process}

An important aspect during the whole development process of a new vehicle, drivetrain system or component is the precise documentation of each step. For calibration engineers, the use of rating systems is a reliable way of documenting 


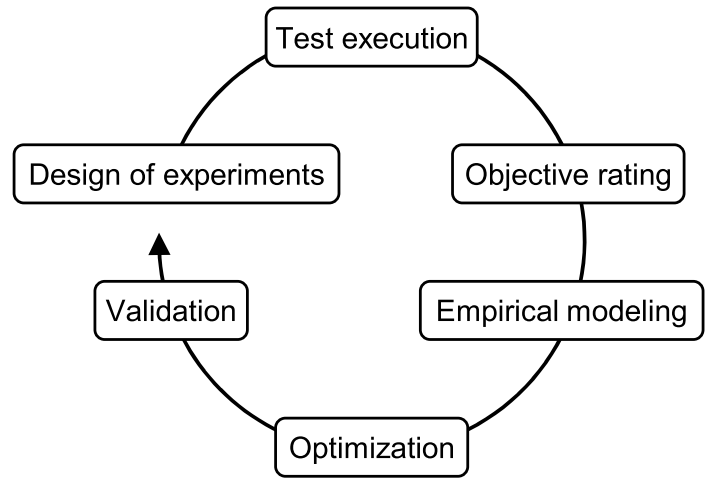

Fig. 5 Optimization process chain using objective ratings

their work. This could be done as presented in this study by the use of graphical plots (see Sect. 3.1) and/or tabular values. Then, this information could be used in a revision process to recapitulate and verify the decisions made.

\subsubsection{Optimization Process}

The calibration of a drivetrain is an iterative process, during which multiple factors have to be considered. Objective rating systems can be one part of a complete toolchain, which helps to find the optimal calibration version. Figure 5 illustrates this process.

First, the design of experiments has to be defined. This includes the amount and quality of the tests. For example, if the goal is to optimize the launch behavior of an ICV, the control parameters used by the ECUs to influence the launch behavior have to be identified. Upper and lower limits, as well as a reasonable number of variations between these limits, have to be specified for all possible combinations of these parameters. The amount of variations depends on available time and resources. The tests could be executed on a test track, roller dynamometer or simulation software. After the tests are executed and evaluated using the objective rating system, a multifactorial empirical model has to be created. Afterward, a suitable optimizer algorithm has to be selected in order to find the best possible outcome (e.g. evolutionary algorithms). At last, the results have to be validated. During the whole optimization process, the objective rating system helps to evaluate hundreds of measurements in a consistent and reliable way.

\section{Launch Maneuver Measurements}

To achieve comparable results, the measurements have to fulfill certain criteria, so the following signals have to be recorded:
- Measurement time $t$

- Vehicle speed (of each wheel) $v$

- Vehicle longitudinal acceleration $a$

- Accelerator pedal position APP

Additionally, the following vehicle information have to be provided:

- Vehicle test mass $m$

- ICE/EM maximum power $P_{\mathrm{ICE} / \mathrm{EM} \text {,max }}$

- ICE/EM maximum torque $T_{\text {ICE/EM,max }}$

During the investigations, the accessible vehicle controller area network has been recorded (including the signals mentioned above, as well as additional signals like engine speed and brake pressure). An acceleration sensor that was mounted onto the front passenger seat's rail provided the longitudinal acceleration signal. This sensor has a sampling rate of $1000 \mathrm{~Hz}$, which is required to achieve reasonable filtering and analysis of the highly dynamic vibrations during the launch.

\subsection{Test Procedure}

The tests have been performed by the same human driver on a flat and paved test track with minimum unevenness. The weather conditions were kept as consistent as possible (no rain, dry road, air temperature between 20 and $25^{\circ} \mathrm{C}$, steady engine oil and coolant temperature). Each vehicle performs about 47 to 70 launch maneuvers. They are distinguished by two parameters: the accelerator pedal gradient $A P P_{\mathrm{g}}$ and the accelerator pedal steady end value $A P P_{\mathrm{s}}$. Figure 6 shows these parameters for four exemplary launch maneuvers that are explained in the following paragraph.

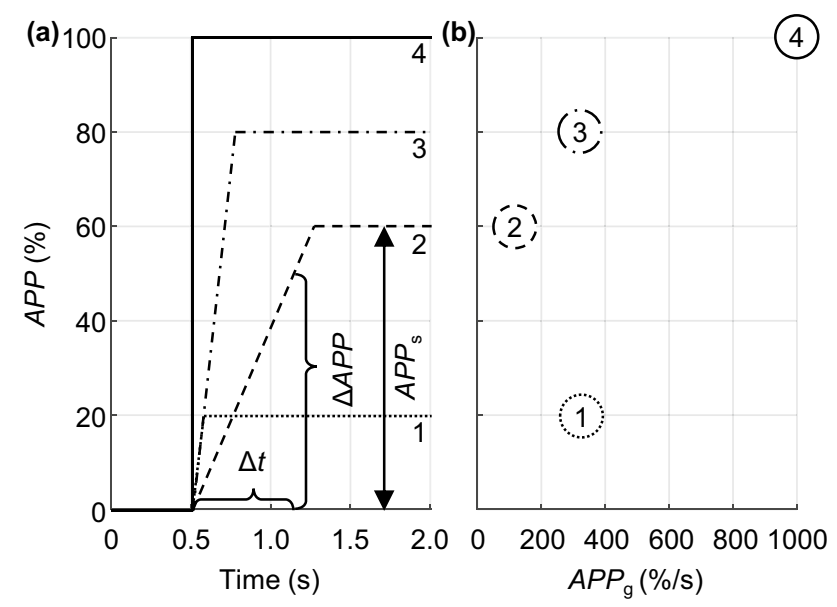

Fig. 6 (a) Exemplary accelerator pedal actuation during the launch phase of four separately performed tests and (b) corresponding scatter plot 


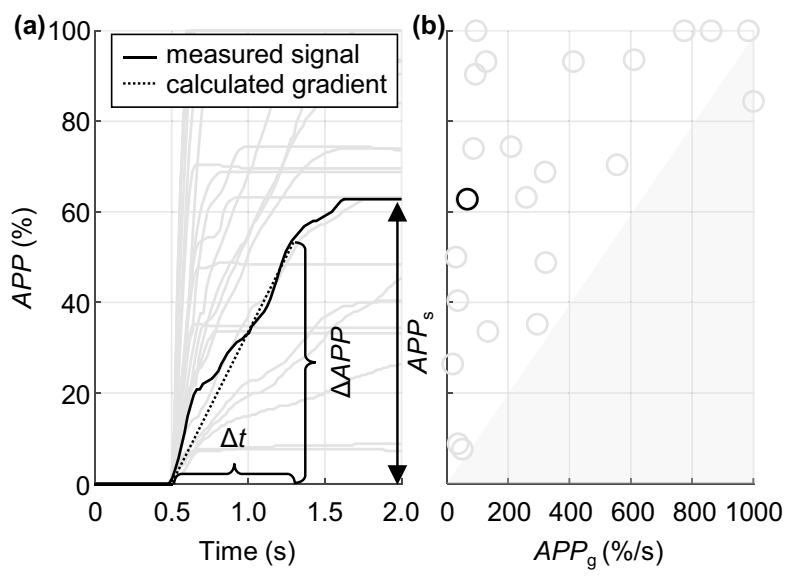

Fig. 7 (a) Measured accelerator pedal actuation and (b) corresponding scatter plot

The accelerator pedal gradient $A P P_{\mathrm{g}}$ is based on the time difference $\Delta t$ and the accelerator pedal position difference $\triangle A P P$, which is defined as $85 \%$ of the accelerator pedal position steady value $A P P_{\mathrm{s}}$.

$A P P_{\mathrm{g}}=\frac{\Delta A P P}{\Delta t}=\frac{0.85 \cdot A P P_{\mathrm{s}}}{\Delta t}$

The factor of $85 \%$ is used to consider the real accelerator pedal actuation behavior (see Fig. 7). After determining $A P P_{\mathrm{s}}$ and $A P P_{\mathrm{g}}$, the respective launch events may be transferred into the map in Fig. 6b. This map is called "fingerprint" and is used to visualize the results of the objective rating (see Sect. 4.1). Each launch is represented by a dot (no. 1 to 4 ). The goal is to cover the fingerprint by 3 different accelerator pedal position gradients (slow, medium, fast) for 10 different end values of accelerator pedal position (10\% to $100 \%)$. Higher coverage is desirable but not necessary. Since the tests are performed by a human driver, the actual accelerator pedal actuation will differ from the given theoretical examples in Fig. 6. Figure 7 a shows the measured accelerator pedal position during the performed tests. Due to the acceleration of the vehicle, the driver cannot keep a constant gradient. The growth rate of the accelerator pedal position flattens until the $A P P$ reaches a steady value $\left(A P P_{\mathrm{s}}\right)$.

Figure $7 \mathrm{~b}$ presents the coverage of the fingerprint. While the upper left half of the fingerprint (white area) includes most of the measurements, the lower right half of the map (gray area) includes only one measurement. For human drivers, it is barely possible to press the accelerator pedal very quickly (high gradient) and then remain at a low accelerator pedal position. A driving robot has the potential to produce higher gradients at the steady end values of low accelerator pedal position, but this requires a test bench like a dynamometer. The steady end value $A P P_{\mathrm{s}}$ is held for at least $3 \mathrm{~s}$ to allow the key parameters $K P_{\text {vib }}$ and $K P_{\text {dis }}$ to be calculated (see Sects. 2.2.2 and 2.2.4). After $3 \mathrm{~s}$, the accelerator pedal may be released. The vehicle has to be stopped completely before the next measurement starts.

\subsection{Test Vehicles}

In total, four vehicles have been tested for this study: two ICVs (vehicles V1 and V2), one PHEV (V3) and one BEV (V4). They all represent different drivetrain concepts but belong to the same vehicle segment $(\mathrm{C}$-segment medium cars) as defined by the European Commission [32]. Table 1 shows important information of the test vehicles.

With the exception of V2, all vehicles feature frontwheel drives (FWD). V2 features an all-wheel drive (AWD) system. There are different types of driving modes selectable by the driver. To keep the results as comparable as possible, the standard modes are selected. The selector lever of each car is set to "D". V1 and V2 feature a start/ stop mode. Although it is an important technology to meet fuel consumption and emission requirements, it also artificially limits the launch quality. In this study, the capability of the actual drivetrain concept and its hardware is investigated. Therefore, the start-stop mode is switched off. V3 offers a P2 PHEV. The vehicle's concept features a permanent switched off ICE (especially at standstill) that will only be turned on if needed (higher power/torque demand than the EM can offer). Furthermore, V3 allows different hybrid modes (e.g., sport mode, pure electric mode and recharge mode). For this study, only the standard hybrid mode is considered. V4 also offers different selectable modes (e.g., eco mode) that influence the power output of the EM. Only the standard driving mode is considered.

Table 1 Test vehicle information

\begin{tabular}{lllll}
\hline Vehicle & V1 & V2 & V3 & V4 \\
\hline Segment & C & C & C & C \\
Drivetrain concept & ICV & ICV & PHEV & BEV \\
Power source & diesel & gasoline & gasoline/electric & electric \\
Driven axle & FWD & AWD & FWD & FWD \\
$P W R(W / k g)$ & 54 & 135 & 88 & 57 \\
$T W R(N m / k g)$ & 0.167 & 0.237 & 0.175 & 0.167 \\
Transmission & DCT & DCT & DCT & single speed \\
\hline
\end{tabular}




\section{Results and Discussion}

In this section, the results of the objective ratings are presented and discussed. As described in Sect. 3.1, fingerprints are used to visualize the objective ratings. Each test vehicle will first be evaluated on its own and then compared to the baseline vehicle V1.

\subsection{Fingerprints}

The color scheme and rating scale of the fingerprints is explained in Sect. 2.1. The colored background is based on a 2-dimensional interpolation of the available data. It helps the viewer to identify areas of special behavior. If the variables $A P P_{\mathrm{s}}$ and $A P P_{\mathrm{g}}$ of multiple measurements are identical or within $0.8 \%$ respectively $16 \% / \mathrm{s}$ deviation, the data points are combined to a pie plot in order to not overlap with each other. Additionally, the mean objective rating $\overline{O R}$ is given in the lower right corner of each fingerprint. This average value is calculated by using the interpolated background data. Therefore, it is not dependent on an evenly distributed amount of measurements.

\subsubsection{Vehicle 1 (ICV, Diesel, FWD)}

V1 is powered by a conventional diesel engine linked to a DCT and using FWD. It represents the baseline drivetrain and will be compared to in Sect. 4.1.5. Figure 8 shows the
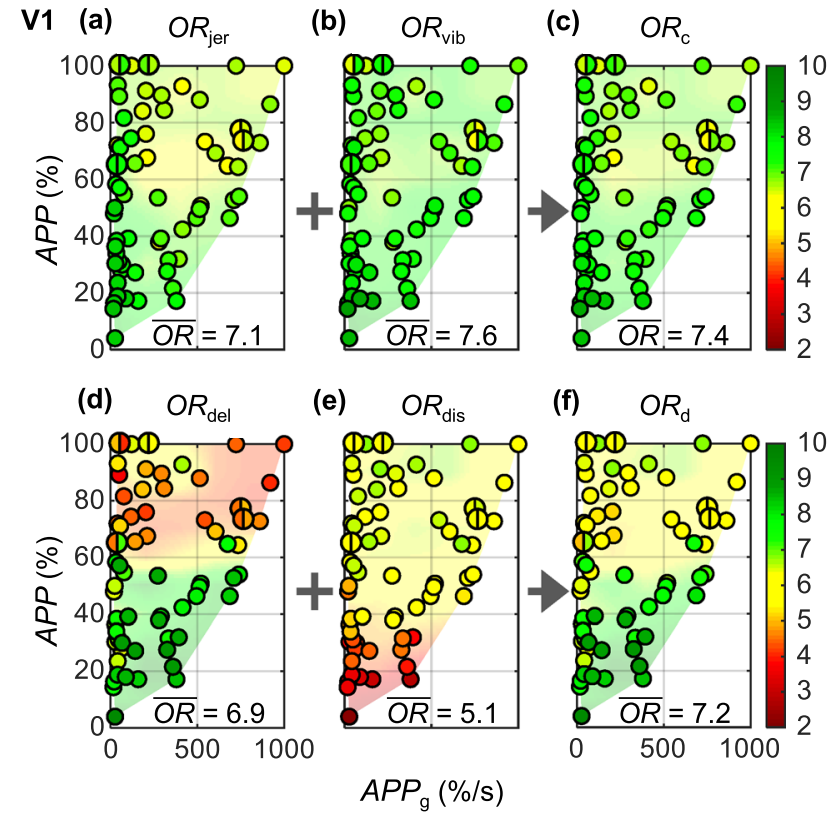

Fig. 8 Objective ratings of vehicle 1 (V1) for the parameters (a) $O R_{\text {jer, }}$, (b) $O R_{\text {vib }}$, (d) $O R_{\text {del }}$, (e) $O R_{\text {dis }}$, as well as the combined ratings (c) $O R_{\mathrm{c}}$ and (f) $O R_{\mathrm{d}}$ objective ratings of 70 measurements for the four key parameters $O R_{\text {jer }}, O R_{\text {vib }}, O R_{\text {del }}$ and $O R_{\text {dis }}$ as well as the combined weighted ratings $O R_{\mathrm{c}}$ and $O R_{\mathrm{d}}$.

The rating $O R_{\text {jer }}$ (Fig. 8a) shows "good" results for low to medium pedal positions; while positions above $60 \%$ result in lower ratings between 6 and 7. $O R_{\text {vib }}$ (Fig. 8b) shows better results in areas of higher positions. The combined comfort rating $O R_{\mathrm{c}}$ (Fig. 8c) shows an average rating based on the weighting coefficients. The dynamic defining parameters $O R_{\text {del }}$ (Fig. 8d) and $O R_{\text {dis }}$ (Fig. 8e) show a more heterogeneous result. While the delay during lower accelerator pedal positions is "good" to "very good", positions higher than $60 \%$ lead to partially "deficient" results. The distance traveled in three seconds is "deficient" in low APP areas up to $30 \%$. Above that, the results are "sufficient" to "satisfactory". The weighting of these two parameters depend on the accelerator pedal position APP. At low pedal positions, the delay is weighted higher and vice versa. Therefore, the resulting combined rating $O R_{\mathrm{d}}$ (Fig. 8f) does not show deficient results.

\subsubsection{Vehicle 2 (ICV, Gasoline, AWD)}

According to Table 1, V2 has a higher power to weight ratio and torque to weight ratio. It also features an AWD. Thus, it is considered a sporty car. The results of 61 measurements in Fig. 9 show some differences compared with V1.

While the comfort-relevant parameters show "deficient" results during and around full load (Fig. 9a-c), the dynamic parameters have higher ratings than V1. Especially, the
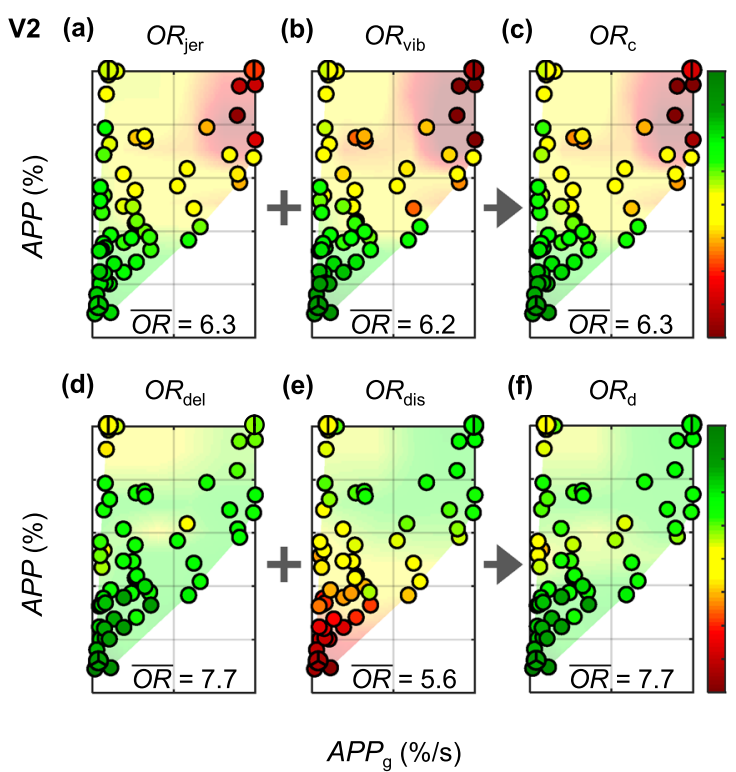

Fig. 9 Objective ratings of vehicle 2 (V2) for the parameters (a) $O R_{\text {jer }}$, (b) $O R_{\text {vib }}$, (d) $O R_{\text {del }}$, (e) $O R_{\text {dis }}$, as well as the combined ratings (c) $O R_{\mathrm{c}}$ and (f) $O R_{\mathrm{d}}$ 
parameter $O R_{\text {del }}$ (Fig. 9d) shows "sufficient" and "satisfactory" behavior for $A P P$ higher than $60 \%$. In addition, the average rating for $O R_{\text {dis }}$ is higher (Fig. 9e) than that for V1. Since the overall rating has a higher weighting on the dynamic rating $O R_{\mathrm{d}}$ (Fig. 9f) for high $A P P$ values, the negative contribution of the comfort rating $O R_{\mathrm{c}}$ is small in this area (see Sect. 4.1.5).

\subsubsection{Vehicle 3 (PHEV, Gasoline/Electric, FWD)}

$\mathrm{V} 3$ is a PHEV with an EM between the gasoline engine and the dual-clutch of the DCT (P2 hybrid). Thus, it represents a combination of conventional and pure electric vehicles. Before each launch, the ICE is switched off and will only start if the torque demand is higher than a certain threshold. Therefore, low accelerator pedal position launches are similar to launches in BEVs. Figure 10 shows the results of 47 measurements for V3. The comfort rating $O R_{\mathrm{c}}$ (Fig. 10c) has the same average value compared to V1. However, while the distribution of $O R_{\text {jer }}$ is similar (Fig. 10a), there is a hot spot around the full-load area of $O R_{\text {vib }}$ (Fig. 10b). As expected for a vehicle with an electric drive unit, the delay $O R_{\mathrm{del}}$ (Fig. 10d) shows the results of "very good", with an average rating of 8.4. On the other hand, the average rating for the distance traveled in three seconds is lower compared to V1 (Fig. 10e) but is more unevenly distributed. High values for $A P P$ over $80 \%$ show "good" results, while low values for $A P P$ up to $40 \%$ show "insufficient" results. The dynamic rating $O R_{\mathrm{d}}$ (Fig. 10f) shows "good" results with an average rating of 7.9.
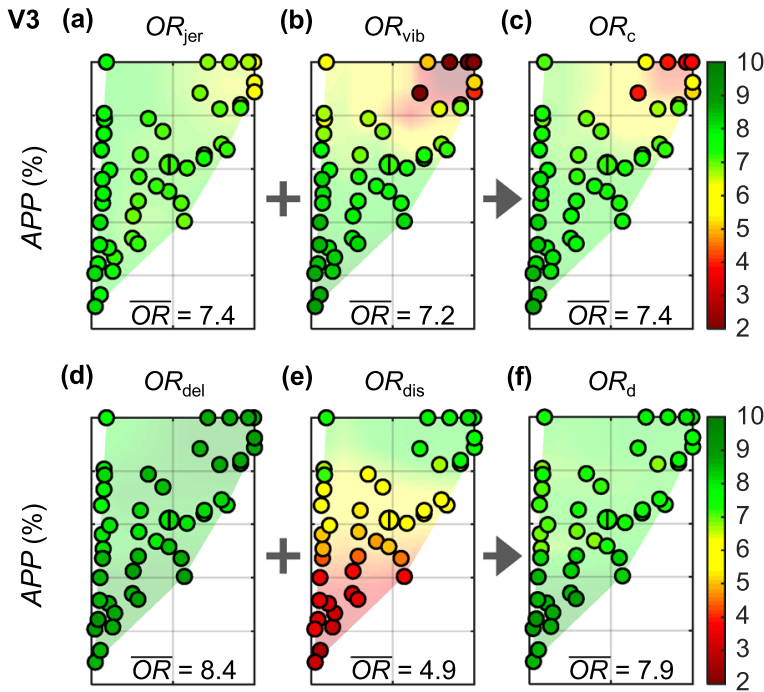

(e) $O R_{\text {dis }}$

(f) $\quad O R_{\mathrm{d}}$

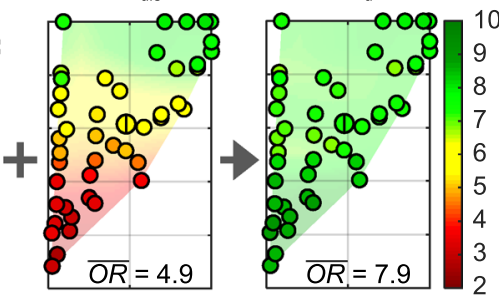

$A P P_{\mathrm{g}}(\% / \mathrm{s})$

Fig. 10 Objective ratings of vehicle 3 (V3) for the parameters (a) $O R_{\text {jer }}$, (b) $O R_{\text {vib }}$, (d) $O R_{\text {del }}$, (e) $O R_{\text {dis }}$, as well as the combined ratings (c) $O R_{\mathrm{c}}$ and (f) $O R_{\mathrm{d}}$

\subsubsection{Vehicle 4 (BEV, Electric, FWD)}

The last vehicle is V4. This BEV features an EM and a single-speed transmission without a launch element. Its power to weight ratio and torque to weight ratio are very similar to that of V1 (Table 1). Figure 11 shows the results of 60 measurements for $\mathrm{V} 4$.

The average comfort-relevant ratings (Fig. 11a-b) are "satisfactory" to "good". During high load, there is a tendency to stronger jerks and vibrations throughout the measurements that lead to medium ratings. The average combined rating $O R_{\mathrm{c}}$ (Fig. 11c) is equal to that of V1 (Fig. 8c). The ratings for $O R_{\mathrm{del}}$ (Fig. 11d) are very close to that for the hybrid vehicle V3 (Fig. 10d). Since the results for $O R_{\text {dis }}$ are "very good" to "excellent" at high values for $A P P$ (Fig. 11e), the average rating $O R_{\mathrm{d}}$ is the highest of all tested vehicles (Fig. 11f).

\subsubsection{Overall Ratings and Difference Plots}

In this section, the overall ratings $O R_{\mathrm{o}}$ are shown for all four test vehicles (Fig. 12). The results are based on Eq. (13). Additionally, Fig. 13 shows the differences of $O R_{\mathrm{o}}$ for V2, V3 and V4 compared to the baseline vehicle V1. Since all vehicles are series cars, it is expectable to have overall ratings above 5. The average rating for $\mathrm{V} 1$ is 7.1 with clear deficits for APP higher than 60\% (Fig. 12a). V2 has slightly better results at higher loads (Fig. 12b). The third vehicle shows a homogenous distribution of ratings with close ratings between 7 and 8 (Fig. 12c). The best overall results are
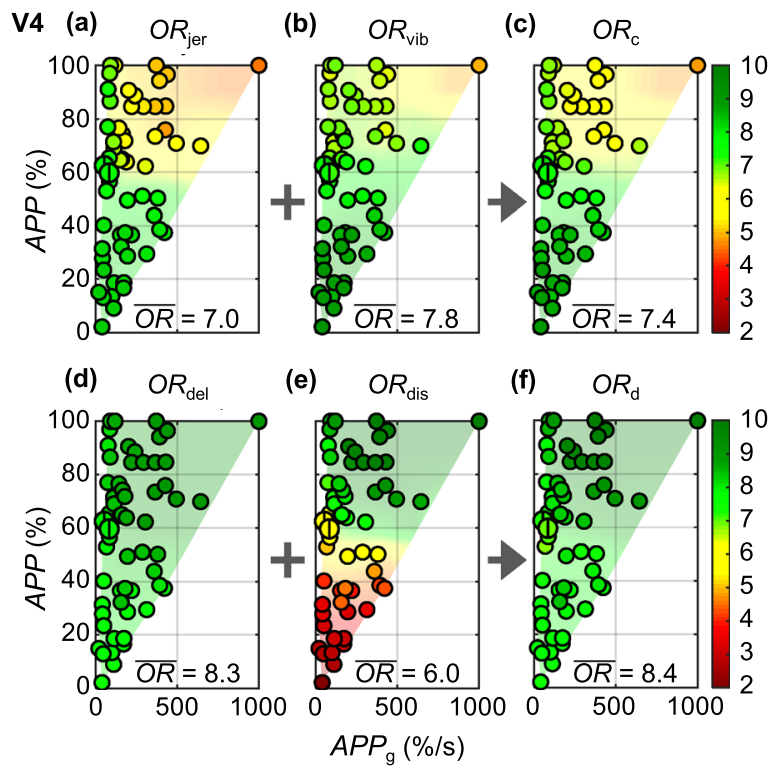

Fig. 11 Objective ratings of vehicle 4 (V4) for the parameters (a) $O R_{\text {jer }}$, (b) $O R_{\text {vib }}$, (d) $O R_{\text {del }}$, (e) $O R_{\text {dis }}$, as well as the combined ratings (c) $O R_{\mathrm{c}}$ and (f) $O R_{\mathrm{d}}$ 


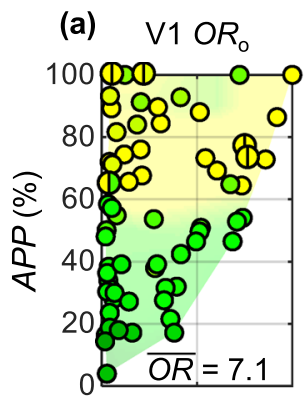

(b) $\mathrm{V} 2 \mathrm{OR}$
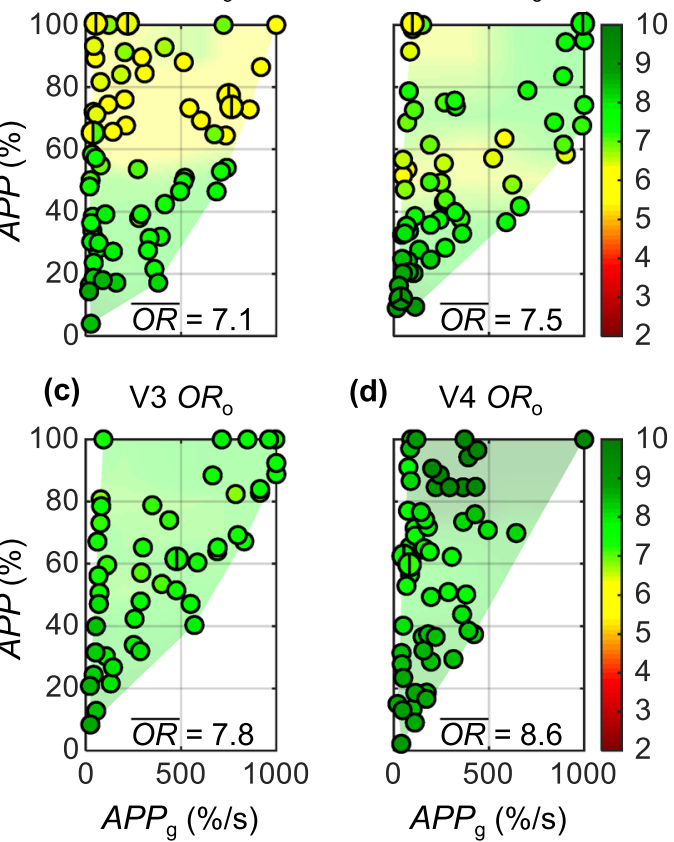

(d)

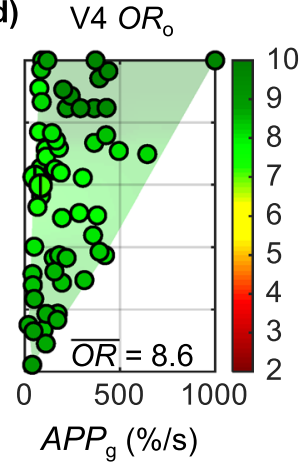

Fig. 12 Overall ratings $O R_{\mathrm{o}}$ for the vehicles (a) V1, (b) V2, (c) V3 and (d) V4

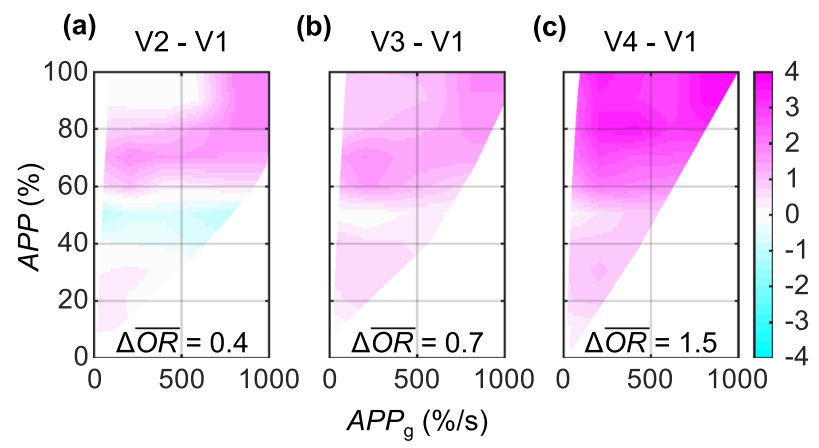

Fig. 13 Difference plots of the overall ratings $O R_{o}$ referred to the baseline vehicle V1 for (a) V2, (b) V3 and (c) V4

found for V4 (Fig. 12d). Especially, the results for high loads are "very good" to "excellent". The average rating is 8.6.

The difference rating $\Delta O R_{\mathrm{V} n, x, y}$ is calculated by subtracting the interpolated data of $\mathrm{V} 1 O R_{\mathrm{V} 1, x, y}$ from the interpolated data of the analyzed vehicle $O R_{\mathrm{V} n, x, y}$ at each data point.

$\Delta O R_{\mathrm{V} n, x, y}=O R_{\mathrm{V} n, x, y}-O R_{\mathrm{V} 1, x, y}$

According to Fig. 13, mainly at higher accelerator pedal positions V1 is outperformed by the other test vehicles. At medium accelerator pedal positions, V2's results are slightly worse (Fig. 13a). V3 shows the same or better ratings with an average improvement of 0.7 grades (Fig. 13b). The clearest differences can be observed for V4 in Fig. 13c. The $\mathrm{BEV}$ performs better ratings with average improvement of 1.5 grades.

\subsection{Discussion}

The results have shown clear advantages of electrified drivetrains regarding the objectively quantified ratings, especially for the dynamic-relevant parameters. However, these electrified vehicles have some potential for improvements. The comfort ratings during high load launches have shown "deficient" (V3) and "borderline" grades (V4). Since the dynamic ratings are more important at high accelerator pedal positions, the overall ratings are not negatively influenced by the comfort ratings. The driver is subconsciously paying more attention toward the performance instead of concentrating on a smooth launch. Comfort and dynamic typically form a trade-off. The improvement of one will often result in the deterioration of the other. However, it is possible to optimize the control parameters of the ICE, EM and transmission ECUs to improve the overall rating. The improvement heavily depends on the initial calibration.

The results provided by the objectification tool can be used for evaluation during an optimization process as described in Sect. 2.3.3. Possible adjustable control parameters for ICVs and PHEVs with DCTs are clutch pressure gradients, clutch slip and the corresponding crankshaft and transmission input shaft speeds. These parameters can be varied in several combinations using fitting optimization algorithms. Closing the clutch while keeping the engine speed low will usually result in a smoother launch but poorer dynamic behavior. Allowing the engine to increase the crankshaft speed before engaging the clutch typically leads to a short delay but also produces higher torque and therefore a higher peak acceleration.

An important task during launches with $\mathrm{P} 2$ hybrids is the restart of the ICE and incorporating it into the power flow by closing the separating clutch without sensible jerks. On the other hand, BEVs do not require clutches and therefore offer less significant adjustable parameters compared to ICVs and PHEVs. Since V4's comfort rating at higher accelerator pedal positions shows some deficits, it could be suggested to smoothen the edges during the torque increase in order to reduce the launch jerk. To perform the described optimization process, complete access to the ECUs has to be provided by the manufacturer. During this study, only CAN data could be recorded. Therefore, no changes could be made to the ECU's control parameters to optimize the launch behavior.

Figure 14 shows a comparison of full-load launches $\left(A P P_{\mathrm{s}}=100 \%\right.$ and $\left.A P P_{\mathrm{g}}=1000 \% / \mathrm{s}\right)$ of each test vehicle. 

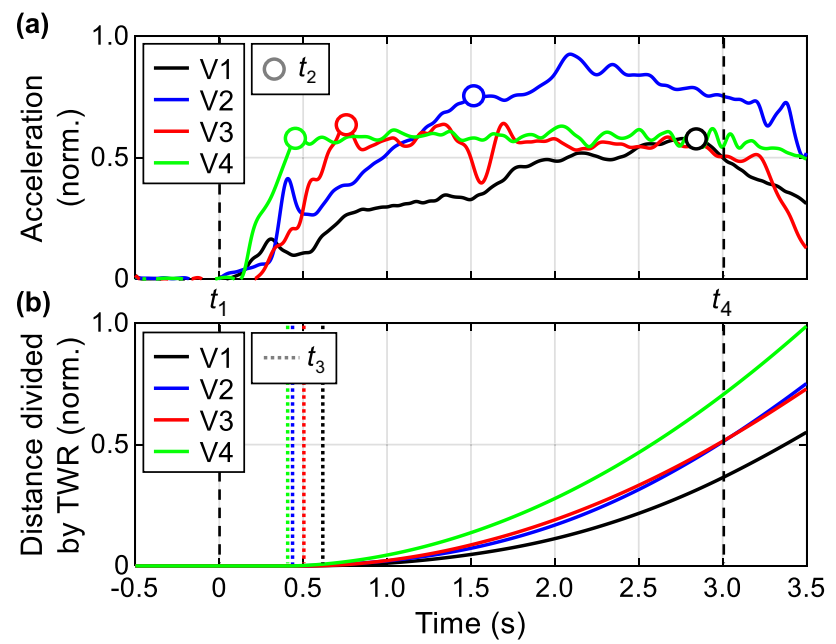

Fig. 14 Measurements of full-load launches of all test vehicles at $A P P_{\mathrm{s}}=100 \%$ and $A P P_{\mathrm{g}}=1000 \% / \mathrm{s}$ showing (a) the normalized longitudinal acceleration as well as the time step $t_{2}$ and (b) the normalized distance divided by the TWR as well as the time step $t_{3}$

The time steps $t_{1}$ to $t_{4}$ are the same as described in Fig. 3. They are used to calculate the key parameters (see Sect. 2.2).

Figure 14a shows the normalized $10 \mathrm{~Hz}$ low-pass filtered acceleration signal measured at the passenger seat's rail. The BEV (V4) reaches its maximum acceleration within less than $0.5 \mathrm{~s}$ with an almost vertical increase. The PHEV (V3) needs approximately $0.3 \mathrm{~s}$ longer with a similar shaped increase. However, at $1.5 \mathrm{~s}$ there is a drop of acceleration resulting from synchronizing the restarted ICE. V2's acceleration reaches a local maximum at $0.4 \mathrm{~s}$ and then drops by $40 \%$. The acceleration starts to increase again and reaches the time step $t_{2} 1.5 \mathrm{~s}$ after pressing the accelerator pedal. The acceleration increases further to its absolute maximum at $2.1 \mathrm{~s}$. V1's acceleration also shows a similar behavior as V2's. Within the first $0.5 \mathrm{~s}$, there is a noticeable oscillation of the signal. A saturated level is reached at $2.9 \mathrm{~s}$. While the maximum acceleration of V1, V3 and V4 are very similar, V2 shows a much higher acceleration. This is realized mainly by the AWD system, which allows a greater traction force to be transmitted to the road. In Fig. 14a, the earlier described potential for optimization is shown. Both electrified vehicles profit from a smoother transition between standstill and maximum acceleration. Additionally, the PHEV shows deficits when engaging the ICE into the power flow that leads to lower comfort ratings. V1's and V2's comfort could also be improved by reducing the oscillation during the first $0.5 \mathrm{~s}$. It is important that these changes do not negatively affect performance.

In Fig. 14b, the normalized distance divided by the TWR according to Eq. 9 is given. The distance traveled after $3 \mathrm{~s}$ shows that with regard to the TWR, V4 achieves the highest result. V2's and V3's results are close to each other while
V1 has the lowest result. Since the TWR of V1 and V4 are identical, this means the distance traveled by V4 is almost twice as far. This demonstrates the benefits of electrified drivetrains compared to turbocharged diesel engines combined with DCTs. The time step $t_{3}$ is also given in Fig. 14b. It is the point of time when the velocity is greater than zero (measured by speed sensors at the wheels). V2 and V4 reach this point almost at the same time. V3 and V1 need $0.1 \mathrm{~s}$ and $0.2 \mathrm{~s}$ longer, respectively. The acceleration during $t_{1}$ to $t_{3}$ is used to calculate $K P_{\text {jer. }}$. The timespan between $t_{1}$ and $t_{4}$ is relevant to $K P_{\text {vib. }}$. Therefore, the early drop of acceleration of $\mathrm{V} 2$ is negatively influencing the $O R_{\text {jer }}$, while the synchronization of the ICE of V3 is negatively influencing the $O R_{\mathrm{vib}}$.

\section{Conclusions}

First, an objective rating system, designed to quantify the perceived quality of vehicle launches, has been introduced. This system has been slightly modified from its original version to be used for electrified drivetrains like PHEVs and BEVs. The study has introduced the calculation of the relevant key parameters and their weighting in order to achieve objective ratings for comfort, dynamic and overall results.

Second, the results have been presented in the form of fingerprints that show the objective ratings for the key parameters and their combinations. The results show clear advantages of electrified drivetrains compared to their conventional counterparts. The average overall rating for the PHEV is 0.7 points higher (10\%) compared to the diesel engine ICV. The BEV shows an even better performance with 1.5 points more $(21 \%)$ than the baseline vehicle. In particular, the dynamic parameters benefit from the quick response of the electric motor. The comfort, especially during high load launches, shows room for optimization.

Lastly, an overview of potential adaptable parameters to optimize the launch behavior has been given. The used methods proved to be valid in comparing conventional and electrified drivetrains. To detect minor differences between pure electric vehicles, a specific objective rating system for this purpose should be designed in the future.

Funding Open Access funding enabled and organized by Projekt DEAL.

\section{Compliance with Ethical Standards}

Conflict of interest On behalf of all the authors, the corresponding author states that there is no conflict of interest.

Open Access This article is licensed under a Creative Commons Attribution 4.0 International License, which permits use, sharing, adaptation, distribution and reproduction in any medium or format, as long 
as you give appropriate credit to the original author(s) and the source, provide a link to the Creative Commons licence, and indicate if changes were made. The images or other third party material in this article are included in the article's Creative Commons licence, unless indicated otherwise in a credit line to the material. If material is not included in the article's Creative Commons licence and your intended use is not permitted by statutory regulation or exceeds the permitted use, you will need to obtain permission directly from the copyright holder. To view a copy of this licence, visit http://creativecommons.org/licenses/by/4.0/.

\section{References}

1. List, H.O., Schoeggl, P.: Objective evaluation of vehicle driveability. SAE Tech. Pap. (1998). https://doi.org/10.4271/980204

2. Dorey, R.E., Holmes, C.B.: Vehicle driveability - its characterisation and measurement. SAE Tech. Pap. 1999-01-0949 (1999). https://doi.org/10.4271/1999-01-0949

3. Wei, X., Rizzoni, G.: Objective metrics of fuel economy, performance and driveability-a review. SAE Tech. Pap. 2004-01-1338 (2004). https://doi.org/10.4271/2004-01-1338

4. Isa, I., Abidin, M., Mansor, S.: Objective driveability: integration of vehicle behavior and subjective feeling into objective assessments. J. Mech. Eng. 6, 782-792 (2014). https://doi.org/10.15282 /jmes.6.2014.6.0076

5. Guse, D., Heusch, C., Pischinger, S., et al.: Objectified drivability evaluation and classification of passenger vehicles in automated longitudinal vehicle drive maneuvers with engine load changes, SAE Tech. Pap. 2019-01-1286, (2019). https://doi. org/10.4271/2019-01-1286

6. Huang, W., Liu, H.J., Ma, Y.F.: Drivability evaluation model using principal component analysis and optimized extreme learning machine. J. Vib. Control 25(16), 2274-2281 (2019)

7. Schoeggl, P., Ramschak, E.: Vehicle driveability assessment using neural networks for development, calibration and quality tests. SAE Tech. Pap. 2000-01-0702 (2000). https://doi. org/10.4271/2000-01-0702

8. Schoeggl, P., Ramschak, E., Bogner, E.: On-board optimization of driveability character depending on driver style by using a new closed loop approach. SAE Tech. Pap. 2001-01-0556 (2001). https ://doi.org/10.4271/2001-01-0556

9. Schoeggl, P., Koegeler, H.M., Gschweitl, K., et al.: Automated EMS calibration using objective driveability assessment and computer aided optimization methods. SAE Tech. Pap. 2002-01-0849 (2002). https://doi.org/10.4271/2002-01-0849

10. Dorey, R.E., Martin, E.J.: Vehicle driveability-the development of an objective methodology. SAE Tech. Pap. 2000-01-1326 (2000). https://doi.org/10.4271/2000-01-1326

11. Everett, R.L.: Measuring vehicle driveability. SAE Tech. Pap. (1971). https://doi.org/10.4271/710137

12. Falk, P., Hubmann, C.: Objective driveability development of motorcycles with AVL-DRIVE. SAE Tech. Pap. 2014-32-0020 (2014). https://doi.org/10.4271/2014-32-0020

13. Wei, X.: Modeling and control of a hybrid electric drivetrain for optimum fuel economy, performance and driveability. Dissertation, The Ohio State University (2004)

14. Shin, C.W., Choi, J., Cha, S.W., et al.: An objective method of driveability evaluation using a simulation model for hybrid electric vehicles. Int. J. Precis. Eng. Manuf. 15, 219-226 (2014). https ://doi.org/10.1007/s12541-014-0328-7
15. Shin, C.W., Kim, H., Kim, M.K., et al.: Development of an evaluation method for quantitative driveability in heavy-duty vehicles. J. Mech. Sci. Technol. 28, 1615-1621 (2014). https://doi. org/10.1007/s 12206-014-0107-5

16. Lakshmanan, S., Palaniappan, A., Chekuri, V.: Methodology for evaluation of drivability attributes in commercial vehicle. SAE Tech. Pap. 2015-01-2767, (2015). https://doi. org/10.4271/2015-01-2767

17. Nehlsen, M., Jedicke, F., Bogner, E., et al.: Driveability analyses on a chassis dynamometer. ATZ Worldw. 108, 9-11 (2006). https ://doi.org/10.1007/BF03224824

18. Zehetner, J., Schoeggl, P., Dank, M., and Meitz, K.: Simulation of driveability in real-time. SAE Tech. Pap. 2009-01-1372 (2009). https://doi.org/10.4271/2009-01-1372

19. Plötner, A., Bilen, Ö., Küçükay, F.: Simulation of the drive-off performance with turbocharged diesel engines. IFAC Proc Volumes 45(30), 146-153 (2012). https://doi.org/10.3182/20121 023-3-FR-4025.00034

20. Dhand, A., Cho, B., Walker, A., et al.: Optimization potential of the vehicle launch performance for start-stop micro-hybrid vehicles. Proc. IMechE 224(8), 1059-1070 (2010). https://doi. org/10.1243/09544070JAUTO1496

21. Cacciatori, E., Bonnet, B., Vaughan, N.D., et al.: Launch and driveability performance enhancement for a parallel hybrid with a torque controlled IVT. SAE Tech. Pap. 2005-01-3831 (2005). https://doi.org/10.4271/2005-01-3831

22. Wehbi, K., Bestle, D., Beilharz, J.: Automatic calibration process for optimal control of clutch engagement during launch. Mech. Based Des. Struct. Mach. 45(4), 507-522 (2017). https://doi. org/10.1080/15397734.2016.1250221

23. Küçükay, F., Lange, A., Li, L.: Highly fuel-efficient transmission and propulsion concepts. Automot. Innov. 1, 35-42 (2018). https ://doi.org/10.1007/s42154-018-0004-1

24. Li, L., Chen, H., Küçükay, F.: Systematic synthesis of dedicated hybrid transmission. Automot. Innov. 2, 231-239 (2019). https:// doi.org/10.1007/s42154-019-00071-3

25. Xu, X., Dong, P., Liu, Y., et al.: Progress in automotive transmission technology. Automot. Innov. 1, 187-210 (2018). https://doi. org/10.1007/s42154-018-0031-y

26. Brendecke, T.: Virtuelle Echtzeitumgebung für Getriebesteuergeräte mit Hardware-in-the-Loop. Dissertation, Technische Universität Braunschweig (2002)

27. Plötner, A.: Objektivierung und Optimierung des Anfahrvorgangs. Dissertation, Technische Universität Braunschweig (2015)

28. Schober, F.: Kalibrierung von Fahrzeuggetrieben im Kundenbetrieb. Dissertation, Technische Universität Braunschweig (2020)

29. Zur zuverlässigen Beurteilung von Fahrzeugen: Aigner. J. ATZ Automobiltechnische Zeitschrift 9, 447-450 (1982)

30. Lerspalungsanti, S., Albers, A., Ott, S., et al.: Human ride comfort prediction of drive train using modeling method based on artificial neural networks. Int. J. Automot. Technol. 16, 153-166 (2015). https://doi.org/10.1007/s12239-015-0017-2

31. Pawellek, T., Liesner, L., Henze, R., et al.: Objectification method for a customer-optimised ACC system configuration. ATZ Worldw. 118, 72-77 (2016). https://doi.org/10.1007/s3831 1-016-0024-6

32. Commission of the European Communities: Case No COMP/M.1406 - HYUNDAI/KIA. Regulation (EEC) No 4064/89 merger procedure (1999) 